\title{
Questions à propos du sacrifice chez les Diola de Basse-Casamance
}

Questions about sacrifice among the Dyola of Basse-Casamance

Odile Journet

\section{(2) OpenEdition}

\section{Journals}

Édition électronique

URL : http://journals.openedition.org/span/418

DOI : 10.4000/span.418

ISSN : 2268-1558

Éditeur

École pratique des hautes études. Sciences humaines

\section{Édition imprimée}

Date de publication : 1 septembre 1979

Pagination : 77-94

ISSN : 0294-7080

\section{Référence électronique}

Odile Journet, "Questions à propos du sacrifice chez les Diola de Basse-Casamance », Systèmes de pensée en Afrique noire [En ligne], 4 | 1979, mis en ligne le 04 juin 2013, consulté le 19 avril 2019. URL http://journals.openedition.org/span/418; DOI : 10.4000/span.418 
Q U E S T I O N S A PROPOS D

$C H E Z$ L E S D I O LA D E BASSE-CASAMANCE par odile doumet.

Dans l'analyse minutieuse qu'il faisait de la religion diola, L. V. Thomas (1) en soulignait le pragmatisme, celui de paysans fermement attachés à leurs rizières, et l'une de ses dimensions principales, la représentation d'un univers comme "siège de multiples dêplacements de forces nourries et nourrissantes" (2). Le sacrifice diola, à l'instar par exemple du sacrifice dogon, semble au coeur de ce processus de manipulation de forces, mettant en jeu le lien entre humains, esprits (ukin) et Dieu. Mais il est aussi lien social et économique, dont 1 'extension varie selon les circonstances, excluant tantôt les un(e)s, tantôt les autres, lien enfin dont l'efficience paraît parfois remise en cause par certains rites échappant au cadre sacrificiel. C'est surtout en vue d'indiquer les axes qui pourraient guider de futures observations, que je tenterai ici de souligner certains aspects du sacrifice diola, en m'appuyant d'une part sur les travaux de L. V. Thomas, de 1'autre sur des observations personnelles, partielles, car recueillies à propos de thèmes adjacents, enfin d'échanges très fructueux avec $M$. Nazaire Ukeyeng Diatta, un abbé diola, qui lui-même s'êtait beaucoup interrogé à ce propos.

1. L. V. THOMAS, Les Diola. Essai d'analyse fonctionnelze sur une population de Basse-Cascononce, IFAN, Dakar, 1959, 821 p.

2. Ibid. p. 792. 
Si les Diola appellent hwasen (huasen) toute offrande aux ukin (3) sous forme végétale ou animale, avec destruction de tout ou partie de la chose offerte, ils accordent néanmoins une importance particulière au sang versé.

1. Nature des offrandes.

Ici, tout dépend bien sûr de l'importance du boekin à qui l'on sacrifie, de l'occasion et du but du sacrifice; mais les offrandes sacrificielles engagent toujours les Diola dans ce qu'ils ont de plus précieux, bétail, riz ou vin de palme.

Du côté animal, l'offrande la plus prestigieuse, c'est le boeuf. A ma connaissance, il s'agit toujours de taureaux, de boeufs ou de gênisses, à l'exception des vaches déjà fécondes. Le sacrifice est d'ailleurs la seule destination des troupeaux de bovins que possèdent les Diola, et qu'ils n'exploitent pas eux-mêmes, si ce n'est pour le fumier. A l'égal des greniers remplis à craquer parfois d'un riz devenu immangeable, les bovins sont le signe par excellence de la richesse, mais aussi du courage et de la dignité : il a fallu, pour les acquérir, travailler durement ses rizières, mais aussi avoir eu, pour épouse, une bonne gestionnaire des provisions accumulées. Ainsi, aux funérailles d'un homme, si l'on exhibe toutes les cornes des animaux sacrifiés par celui-ci (quitte à en emprunter pour 1 'occasion), si, dans les louanges, le surnom "ô toi dont les cornes sont nombreuses" est des plus laudatifs, 1 'on dit que c'est "sa femme qui (les) a achetés".

Le sacrifice d'un ou plusieurs bovins est ainsi requis :

-- Lors des funêrailles d'un homme ou d'une femme adultes, à deux reprises, sans quoi le défunt ne pourra parvenir au monde des ancêtres.

3. Le boekin (plur. ukin) est à la fois le sanctuaire, l'autel, et le gênie qui sert d'intermédiaire aux hommes, face à un Dieu (Ata-Emit ou Emitay), jugé trop lointain. 
L'animal (il peut $y$ en avoir plusieurs, lors du décès d'un vieillard) est immolé dans la cour de la concession natale du défunt, ou dans celle du propriétaire de la bête, d'abord au moment du décès, puis lors d'une fête de "commémoration" (c'est le kasinteen).

-- Lors des fêtes de circoncision, au boekin de circoncision et aux ukin de protection familiale.

- Lors des initiations aux ukin royaux (en plus des chèvres, objets par excellence du sacrifice royal).

-- Très souvent, lors de la création d'un nouveau sanctuaire à partir de I'autel-mâ̂tre.

-- Lors des cérémonies d'intronisation des grands prêtres et prêtresses des associations cultuelles les plus importantes.

-- En cas de meurtre d'un homme : 6 taureaux ou 6 génisses, acquis en échange des rizières confisquées au criminel, sont offerts aux principaux ukin du village, dont l'Elung (contre le vol et le meurtre), plus un autre, offert par la famille du coupable (4).

-- En cas d'abattage d'un boeuf ou d'une vache en brousse ou dans les rizières : la reparation exige le sacrifice de trois bovins, ou, au minimum, d'une génisse et de deux porcs.

-- En cas de sécheresse enfin, aux principaux ukin de pluie (Husila, Earmiman, Ehunya), ce sont alors, de prëférence, des taureaux noirs.

Ces sacrifices, lors des cérémonies collectives, prennent souvent la dimension de véritables potlach : en 1957, L. V. Thomas dénombrait quelque 700 boeufs sacrifiés à l'occasion des fêtes de circoncision dans le village de Kanobion; en 1965, dans le village de Niomoun, 200 boeufs furent abattus lors des fêtes qui réunissaient environ 4000 personnes, ce qui fut jugé faible. De même, à la mort d'un "roi" (oeyi), pratiquement tous ses boeufs sont immolës aux ukin qu'il desservait.

Puis, viennent les chèvres, qui occupent une place privilégiée dans tous les sacrifices aux ukin royaux (5), alors qu'elles restent

4. L. V. THOMAS, op. cit. p. 689.

5. Sur I'institution royale diola, cf. L. V. THOMAS et J. GIRARD, Genese du pouvoix charismatique en Basse-Casamance, IFAN, Dakar, 1969. 
la plupart du temps absentes des autres sacrifices, car objets d'interdits alimentaires très stricts : la consommation de la viande de chèvre comporte toujours le risque d'une atteinte à la fertilité ou à la virilité, par le biais de maladies vénériennes.

Les porcs qui, en maintes occasion, peuvent remplacer les boeufs, sont exigés lors de sacrifices d'une relative importance, au boekin de circoncision, aux ukin royaux (sauf le Jananande, apparu aux hommes sous la forme d'un hippopotame), au KaZahay (boekin de la maternité), etc.

Quant aux poules, poulets et coqs, d'usage courant sur les autels familiaux et lignagers, ils peuvent être de tous les sacrifices. Il ne semble pas -- est-ce seulement le fait d'un regard distrait ? -- que les Diola différencient aussi finement, ou revêtent de signes aussi spécifiques les poulets que, par exemple, les Gourmantché, dont nous parle M. Cartry. La couleur ici semble être un élément déterminant dans le choix de l'animal :

-- poulet blanc sur 1'autel des ancêtres lignagers et familiaux, lors de la présentation du nouveau-né, et lors des principaux événements de la vie familiale;

-- coq rouge aux ukin "parlants" (divination), au boekin de circoncision, lors des funérailles de certains prêtres;

-- poulets de toutes sortes aux funérailles, apparemment sans couleur particulière.

Les moutons n'intervenaient jamais dans 1 'ancien culte des ukin, les Diola ne les élevant pas d'ailleurs. Ils sont actuellement sacrifiés au seul boekin tusiza, boekin de pluie, apparu à une femme, Alinsitwe, dans les années 1940, et dont le culte regroupe diverses ethnies de la région.

Le chien n'est pratiquement plus sacrifié. Mais les récits des premiers observateurs étrangers laissent à penser qu'il servit couramment de victime : c'est le seul cas où le sang de 1'animal pouvait être bu par le sacrifiant, cela lors des funérailles (6).

6. L. V. THOMAS, op. cit. p. 671 . 
Quant aux victimes humaines, certains ukin, particulièrement redoutables, Elung, Jananande, par exemple, pouvaient en exiger périodiquement.

Il faut ici aussi constater qu'aucun des animaux cités n'est mis à mort en dehors du cadre sacrificiel, si du moins l'on intègre à celui-ci les mises à mort effectuées lors des funérailles, ou encore dans le cadre des sociétés de travail, à leur boekin protecteur. Lors des funérailles, tout le monde profite de l'occasion pour tuer de très nombreux poulets "en 1'honneur du mort", ou "aux ancêtres", à seule fin de pouvoir en manger... Quant aux animaux gagnês en rêtribution du travail exécuté par telle ou telle sociêté, ils ne peuvent être abattus n'importe où, mais seulement au boekin (fréquenment figurê par un bâton entouré de fibres de rônier).

Tout huasen, avec ou sans mise à mort d'un animal, comporte de manière générale, libations de vin de palme, voire d'eau, libations auxquelles peuvent se réduire; par exemple, les huasen offerts par une fenme stérile à certains ukin, ou les huasen quasi-quotidiens sur les autels familiaux.

Au centre des préoccupations matérielles et spirituelles des Diola, seule nourriture digne de ce nom, symbole de la richesse, le riz est une offrande de choix. Conservé dans les greniers en gerbes, il n'est jamais offert tel quel, mais sous forme de grains, détachés par le sacrifiant ou le prêtre et jetés sur 1'autel, ou encore, sous forme de farine. Une pratique courante est d'en jeter une partie sur 1'aute1, de malaxer le reste pour en faire des boulettes qui seront partagées entre les participants. On la retrouve plus spécialement lors de la consécration des enfants aux ukin familiaux, lors des sacrifices pour les rêcoltes, aux ukin des fenmes, Ehmnya et KaZahay. Le kasara, sacrifice de riz en demande ou remerciement de bonnes récoltes, comporte division, mẻlange, puis redistribution des galettes préparées par toutes les femmes du village, avec de la farine, de l'eau et du sucre, avant qu'une partie en soit offerte aux ukin protecteurs (7). Chez les Diola Bandial. (sur la rive sud de la Casamance), toute la production

7. L.V. THOMAS, op. cit. p. 737 . 
d'une rizière royale est destinée aux offrandes pour obtenir la pluie; c'est cette rizière que 1 'on repique la première. Puis, au début de la récolte, chaque homme doit apporter une gerbe au boekin de culture, avec du vin de palme, à l'annonce du desservant de ce boekin. Celui qui y manquerait, ou entreprendrait ses récoltes avant, risquerait de voir ses rizières se dessécher, et d'être lui-même la proie du boekin (8).

Le riz, lorsqu'il est offert sous forme de farine, est en général pilè à la maison. Mais il est toujours 1'objet d'une manipulation ou d'une prêparation particulière dans le sanctuaire : préparation de boulettes, assemblées sur un mode, repartagées sur un autre. Ces traitements rituels de la graine et de la farine relèveraient encore d'observations plus approfondies.

; Tous les êléments offerts aux ukin, d'origine animale (pour ce qui est du sang et du foie essentiellement) ou végétale, ont en commun d'être pensés, par les Diola, comme "lumineux", véhicules d'énergie vitale, s'opposant par là à certains éléments comme les cheveux, eux conisidérés comme "sombres", porteurs d'énergie de mort. Ce qui pourrait ouvrir ici sur d'autres investigations, à partir de l'examen de tous les cas où les cheveux sont rasés (lors de passages dangereux, naissance, initiation par exemple), et où l'explication par la "purification" n'est peut-être pas toujours pleinement satisfaisante.

2. Quelques aspects du déroulement des opérations.

Les rites prẹparatoires sont peu dêveloppés dans le sacrifice dio1a. Le (la) desservant(e) du boekin, 1'aloemba, vient un peu avant le sacrifice, "préparer le boekin", vérifier et disposer les objets rituels (couteaux, calebasses, bâtons), voire procéder à des libations préalables. Lors du sacrifice, l'aloemba porte souvent un pagne rouge ceint

8. Cf. F. G. SNYDER, L'évolution du droit foncier de Basse-Casamance, Thèse pour le doctorat de IIle cycle, Univ. Paris I, 1973. 
sur les reins. Du côté du sacrifiant, les prêliminaires se rêduisent souvent à une présentation des offrandes, accompagnêe de sa première demande formulêe à d.'intention du desservant, demande qui sera reformulée une fois que ce dernier aura juge l'offrancie recevable. he recours à des procédés divinatoires préalables au sacrifice tr'a rien d'obligatoire. Il intervient, par boekin "parlant" (9), ou encure par voie de songe, lorsque le sacrifiant recherche quel bogkin il a pu offenser, ou lequel l'a "pris", et correlativement, l'indication de 1 'offrande à fournir.

Le sacrificateur (owasen), dans la plupart des cas, est l'atoemba lui-même. Cependant, les deux personnages sont distincts, lorsqu'il s'agit, par exemple, de sacrifier de gros animaux aux ukin féminins, la desservante de L'Finingy ou du Kolariay pouvant elle-même cependant sacrifier les poulets, voire, mais 1 'indication reste à confirmer, dans certains cas, les porcs. Dans les cas où elle ne sacrifie pas ellemême, c'est à un homme de sa famille qu'elle a recours. Qui exactement? Cela reste à préciser. Par ailleurs, lors de cérémonies présidées par un des rois diola, ce sont souvent des prêtres desservant tél cu tol boekin "coiffé". par l'institution royale qui sacrifient à sa place.

Le sacrifice s'effectue en général dans le sanctuaire, que 1'aute 1 se trouve dans la concession, sur la piace du village, dans la forêt, selon le boekin ou selon la circonstance. S'il s'agit d'un sacrifice animal, il importe seulement qu'un peu de sang coule sur l'autel, ou, en cas d'offrande végêtale, qu'il y ait libation de vin, d'eau, et que l'on jette du riz sur cet autel. Ceci dit, toutes les operations afférentes au sacrifice ne se déroulent pas forcément dans le sanctuaire. La victime peut être dẹplacêe d'une concession à une autre, conme c'est le cas lors des funérailles, lorsqu'on emmène le boenf, dont les neveux utêrins du défunt ont sectionné les jarrets, de la concession de ce dernier à celle du parent ou de l'ani qui a offert l'animal. Après 1'immolation, un peu de sang sera rapporté sur l'autel des ancêtres

9. Le boekin "par1ant", après sacrifice, répond à la demande du patient, par 1'intermëdiaire d'une petite caisse de résonance, d'où sortent des fibres que le prêtre frotte les unes contre les autres, voire encore, par la voix même du prêtre qui parle entre ses dents, L. V. THoMAS, op. cit. p. 758. 
de la concession du mort. C'est ainsi, lors des funérailles, qu'interviennent d'autres techniques de mise à mort que celle de l'égorgement simple, le plus couramment pratiqué : au cours d'une danse, les neveux utérins sectionnent d'un coup net les jarrets de l'animal, ou bien le transpercent de flèches ou de lances, avant l'immolation définitive (par égorgement), tandis que beaucoup de ceux qui assistent à la cérémonie assomment des poulets sur la claie de portage du défunt.

Mais revenons au déroulement même des opérations : une fois mise au point la demande du sacrifiant, le sacrificateur explique les raisons du sacrifice à la victime, puis, tout en demandant aux prêtres défunts de l'assister, il s'adresse au boekin, à l'aide de formules rituelles (10).

C'est bien ici aussi la parole de l'aloemba qui fait l'efficience du sacrifice. Ce circuit de parole, d'après les informations rapportêes par L. V. Thomas, se poursuit par un dialogue entre le boekin et Dieu (Emitay). Lorsqu'il s'agit d'un boekin "parlant", la réponse d'Emitay au boekin est retransmise au sacrifiant par 1'intermédiaire du prêtre -- rêponse immédiate, ou bien différée, dans un songe qui vient au prêtre (cas des ukin familiaux d'interrogation).

Quelles que soient les occasions du sacrifice, les demandes, les offrandes, le schêma est à peu près le même.

3. Destination finale des offrandes.

Dans le cas d'un sacrifice animal, le sang est entièrement versé : c'est la part du boekin ou des ancêtres (?). Le sang n'est jamais bu, à 1 'exception de celui du chien autrefois sacrifié aux funérailles : ceux qui pouvaient prétendre avoir été aussi courageux que le mort pouvaient en boire.

Quant à la viande, plusieurs cas se présentent : elle peut être

10. L. V. THOMAS, op. cit. p. 678. 
Questions à propos du sacrifice chez les Diola de Basse-Casamance 85

abandonnée sur place ou enterrée, comme c'est le cas lors de sacrifices à certains ukin royaux (Jananande), ou lors de 1a "prise" d'un boekin de protection personnel (bakulem) ; rappelons le cas particulier du coq rouge égorgé puis jeté dans le feu lors des rites de circoncision (11). Soit elle est consommée par l'aloemba lui-même, lors de sacrifices demandés par un individu à des fins privees, ou bien lors de sacrifices périodiques que le prêtre doit à son boekin. Soit encore, c'est la figure générale, elle est consommée par l'ensemble de ceux qui sont initiés à la pratique du boekin. Ainsi, seules les femmes de 1'Ehunya (les femmes qui ont au moins un enfant vivant) peuvent consommer la viande des animaux tués à leur boekin. Du côtē masculin, de même, seul le circoncis pourra manger la viande du sacrifice au boekin de circoncision, seul l'initiê à l'enterrement mangera la viande sacrificielıe au boekin afférent... Soit encore, la consomation est permise à tout le monde, lorsque le sacrifice vise un effet valable pour tout le groupe : repas publics à l'échelle du quartier, du village.

Les mâchoires, crânes et tibias restent, quant à eux, exposês dans le sanctuaire; les cornes des bovins vont à leur propriétaire. On retrouvera les mêmes variantes dans la consomation des offrandes végétales.

La prêparation culinaire des produits sacrificiels diffère peu, apparement, des techniques quotidiennes, ce qui nous situe un peu à l'inverse de l'exemple des Mofu, étudiés par J.-F. Vincent, indifférents à la puissance symbolique du sang, mais pratiquant une cuisine rituelle très élaborée (12).

Du point de vue de la consomation des offrandes, les funérailles, encore une fois, vont présenter un cas un peu particulier : comme on 1 'a vu, tout le monde profite de l'occasion pour tuer et manger des poulets. Le riz offert est mangé par la famille du défunt, et particulièrement par les femes nées dans ia concession qui composent les chants funèbres ( $\mathrm{riz}$ alors consommé en grains, jamais en farine, l'interdit inverse s'observant pour la nouvelle accouchëe). Quant au boeuf,

11. L. V. THOMAS, op. eit. p. 697.

12. J.-F. VINCENT, syetemes de pensée en Afrique noire, le sacmifice 1. 1976 . 
il apparaît conme révélateur des relations entre lignées paternelle et maternelle : offert par les frères, fils ou ami du défunt, par le mari, s'il s'agit d'une femme, il ne peut être consommé ni par le père ou la mère, ni par le veuf ou la veuve du mort. S'il est offert par un ami du défunt, les principaux morceaux reviendront à celui-ci et à sa famille, après en avoir donné une part (une cuisse arrière par exemple) à la famille du défunt ; les neveux utérins de l'ami récupèrent souvent le cou et le foie, alors que la tête va plutôt au doyen de sa concession, le poitrail à ses compagnons d'âge... Si c'est un frère ou un cousin du mort qui offre la bête, l'essentiel de la viande reviendra à un ami de ce frère ou cousin. Si c'est un gendre du mort, il repartira lui aussi avec une grande partie de la viande de l'animal sacrifié, mais, en plus, les parents du đéfunt doivent lui fournir un autre boeuf. Le pancréas revient souvent à celui qui a gardé l'animal, tandis que la queue et les cornes, selon les cas, sont données au sacrificateur, à celui qui a élevê 1'animal, ou encore à la famille du défunt. Dans tous les cas, les funérailles sont le moment de prestations des oncles paternels, des frères et fils du mort, aux oncles maternels : pagnes, chèvres, porcs, parfois un boeuf, comme chez les Diola Dyiwat, qui constituent en que1que sorte pour les maternels la contrepartie de la mort de leur neveu ("voleur rituel" (13), mais aussi chargé d'un certain nombre d'obligations rituelles chez son oncle maternel, dont les moindres ne sont pas les tâches funéraires qui lui incombent : laver, habiller, porter la dépouille lors de l'interrogatoire, l'ensevelir, dégager la tombe...). Pour les fils, ces prestations constitueraient plutôt la manifestation de leur innocence dans la:mort de leur père.

Lors des funérailles, le schéma idéal serait la destruction de tout ce qui a appartenu au mort, maison, troupeaux, etc., schéma qui rencontrera, dans la pratique, des difficultés, lorsque les biens en question, troupeaux entre autres, constituent un patrimoine familial, plutôt qu'individue1. La destruction, et la consommation collective, restent cependant chez les Diola le moyen le plus évident d'absorber ces tensions

13. Tel qu'il apparait chez les Thonga, par exemple. Cf: L. de HEUSCH, La dette sacrêe de 1 'oncle maternel, in : Systèmes de signes, mélanges offerts en hommage à Germaine Dieterlen, Paris, Hermann, 1978. 
Questions à propos du sacrifice chez les Diola de Basse-Casamance 87

entre attachement individuel d'une personne à des biens, et propriété collective.

S'agit-il vraiment ici de sacrifice au sens de rite d'union entre le sacré et le profane, comme le définissent Hubert et Mauss ? I1 s'agit ici de "nourrir 1'âme" du mort, de lui permettre de rejoindre en paix le monde des ancêtres, du moins de l'au-delà d'où il pourra éventuellement se réincarner, de lui éviter le triste sort de revenant. On ne s'adresse pas à Dieu ni vraiment au boek̉in. Ce sont les ancêtres que I'on invoque surtout sur l'autel du Kuhuizung dans la concession; mais il est vrai qu'ancêtres et ukin vivent en proximité...

Avant d'essayer de dégager quelques significations, du moins procédés efficaces à l'oeuvre cans le sacrifice diola, on peut tenter d'en regrouper et distinguer les principales occasions :

-- lors d'un passage réputé difficile ou dangereux : initiations, intronisations, consëcration de l'enfant, funérailles, etc. ;

-- en cas de danger ou de menace mortelle à I'égard du groupe ou de l'individu, danger interprété come la conséquence d'une offense volontaire ou involontaire à un boekin : épidémie, sécheresse, maladie, stérilité... ;

-- en vue de se concilier les ukin, avant d'entreprendre travaux, voyage... ;

-- pour satisfaire et. "nourrir" përiodiquement le boekin.

\section{Quelques interprétations possibies.}

Le procédé par lequel opère le sacrifice, tel que les Diola se le representent, est très comparable à celui qui est à l'oeuvre dans le sacrifice dogon par exemple : c'est bien ici un processus de circulation d'énergie vitale ( $y_{\hat{\imath}}$ ), libérée par le sacrifice et vêhiculée par certains élêments privilégiés, qui doit profiter à son terme à celui (celle, ceux) en faveur de qui est offert le sacrifice. Cette circulation est rendue efficiente par la parole du (ce la) prêtre(sse) alertant le boekir.

I. semble également que I'on retruuve chez les Diola cette idée exprimée par G. Dieterlen (14) que, dans le cas d'une faute par rupture 
d'interdit, le problème n'est pas d'effacer une souillure, un "en-trop", mais bien plutôt de supplêer à un manque d'énergie vitale. Quelques indications permettraient d'aller dans ce sens : ainsi, lors de certains rituels, dits de "purification", on parle plutôt de "rafraîchir" la personne; or le malheur et la mort sont considérés comme quelque chose de chaud (quand on fait un cauchemar, mauvais présage, on verse de l'eau sur le sevil de la porte pour le refroidir et empêcher le malheur d'entrer). Le revenant, lui qui est en dêfaut complet d'énergie vitale, exclu du monde des vivants, comme de celui des morts, est un personnage qui a du feu plein la tête et le corps. On pourrait done faire l'hypothèse que la libation sacrificielle permet également de "rafraîchir", c'est-à-dire, en écartant l'énergie de mort, de compléter, de renforcer 1 'énergie vitale.

Là, cependant, s'arrête le rapprochement avec l'exemple dogon, Ie sacrifice diola ne comportant pas la réactualisation de la mise à mort d'un héros mythique.

Si le sacrifice est libëration d'ènergie vitale, il suppose bien mort quelque part. Cette mort du sacrifice, dans les conceptions diola, doit être renouvelée tout au long de la vie d'un individu : l'univers diola semble en effet s'organiser autour $d$ 'un axe qui relie un monde de ténèbres (le nôtre) à un monde de lumière, lequel serait celui des ancêtres, des morts accomplis, pour lesquels tous le rites funéraires ont été achevés. Entre ces deux pôles, qui ne sont pas nettement séparês, existe un monde de pénombre, monde aquatique, où sẻjournent les ahuka ( $($ hriwa), ceux qui sont à naître. Chaque moment privilégié de 1'existence, naissance, initiation, mort, exprime de manière plus aigue cette tension, ce passage progressif d'un monde encore imprëgné d'une présence de mort à un état de vie plus intense. Ce sont précisément ces éléments sacrifiês, "lumineux", qui doivent aider à ce passage, jusqu'au sacrifice final du kasinten, qui a lieu quelques mois ou quelques années après le dêcès, et permet, 1' intégration du mort "accompli" au monde des ancêtres.

14. CF. G. DIETERLEN, Introduction à de nouvelles recherches sur le sacrifice chez les Dogon, Systèmes de pensée en Afrique noire, Le sacrifice 1, 1976. 
Mais il n'y a pas que l'animal, ou 1 'offrande vêgétale, qui soient en jeu : un certain nombre de faits, et l'exégèse diola elle-même, invitent à remonter de la chose sacrifiée au desservant même du boekin. Les rituels d'intronisation des grands azoembe reproduisent, pour une bonne part, les rites funèbres; c'est à ce moment, entre autres, que circulent les prestations diverses (pagnes, vin de palme...) requises lors des funérailles habituelles. Il semble qu'autrefois les desservants des ukin les plus importants aient ëgalement été tenus au cêlibat, du moins à l'abstinence sexuelle. Tout au long de leur vie, ils restent soumis à un certain nombre d'obligations, dont celle de sacrifier régulièrement et conséquemment à leur boekin. De fait, aujourd'hui comme autrefois, personne ne tient à être responsable d'un culte, même familial, et l'on s'y résigne bien plutôt qu'on y aspire, ce, malgré les apparents avantages alimentaires et économiques que peut procurer cette fonction. Retrouverait-on ici aussi cette idée formulée par I. de Garine, à propos des Moussey, que "le sacrifice use" (15) ?

la dialectique encre énergies de mort et de vie, la puissance symbolique attachée au sang, nous amènent à envisager un rapprochement possibie entre sacrifice, d'une part, moments particuliers lors desquels le sang coule, et enfin d'autres rites non sacrificiels, mais dont l'efficace peut être comparable.

\section{Cas de l'accouchement.}

Le sang de 1'accouchēe est très fortement chargê d'interdits : il ne peut couler n'importe où, mais seulement dans le lieu bien circonscrit de la "maternité", laquelle se trouvait traditionnellement dans lá forêt, au pied d'un fromager, dissimulée aux regards par des tapades. Jamais une fenme n'accouche dans sa maison, ou dans sa concession, au risque de provoquer les pires calamités; si néanmoins elle se laisse surprendre, que quelques gouttes de sang seulement s'écoulent dans

15. Exposé au L.A. 221, 16 novembre 1978. 
la maison, celle-ci tombe immédiatement sous la coupe du boekin Katahay. Tout ce qu'elle contient (riz, pagnes ustensiles...) est emporté par le boekin, à la suite de quoi la maison est brûlée. Si la femme n'a fait que perdre les eaux, la maison n'est pas brûlée, mais la prêtresse emporte un peu de tout ce qui s'y trouve. Le sang de l'accouchée "contamine" en quelque sorte tout ce qui touche ou entre dans la maternité, ne pouvant plus en ressortir (nourriture, canaris, habits...). Supposé véhicule d'une ênergie qui doit profiter à l'enfant (et à tous les enfants à naître), ce sang ne peut être dispersê, répandu n'importe où, à l'instar du sang sacrificiel. Mais des questions demeurent : quel rôle exact joue la femme ? Peut-on dire qu'elie meurt en quelque sorte pour que son enfant vive ? S'impose aussi à I'idée l'hypothèse de M. Cartry, à propos du sacrifice gourmantché : l'immolation reproduisant le geste de la sage-femme, lorsqu'elle sépare l'enfant de son placenta, ou encore la manière dont les Bouriates (16) désignent le sacrifice : "sacrifice au lieú d'enterrement du placenta".

Mais, chez les Diola, le placenta, enterré dans la maternité, à ma connaissance, n'est pas en tant que tel objet d'attention ou de rites plus particuliers. L'attitude requise de la mère, en tout cas, semblable d'ailleurs à celle qui est exigée du circoncis, souligne cette. "mort des sens" par laquelle elle doit passer. Brusquée, secouée, si les choses ne vont pas assez vite, la mère doit "serrer les dents" et ne rien dire.

Un autre rite pourrait peut-être servir de guide : lorsque la mère meurt en couches, le mari doit apporter à la prêtresse du Kalahay un porc, six poulets et six gerbes de riz qui seront sacrifiés au boekin. Mais tout d'abord elle procède à 1'extraction du foetus, avec une sorte d'acharnement forcé (comme s'il fallait prolonger, souligner la mort de la mère ?). Hubert et Mauss disent à un moment que le sacrifice doit avoir une parfaite continuité : "A partir du moment où il est commencé, il doit se poursuivre jusqu'au bout, et dans une parfaite continuité. Les forces qui sont en action, si elles ne se dirigent

16. M. CARTRY, Le statut de 1'animal dans le système sacrificiel des Gourmantchë, Systèmes de pensée en Afrique noire, cahier 3, Le sacrifice 2, 1978. R. HAMAYON, Marchandage d'âmes entre vivants et morts, ibid. 
pas exactement dans le sens prescrit, èchappent au sacrifiant et au prêtre, et se retournent contre eux, terribies." Cette idée pourrait peut-être guider d'autres observations plus approfondies dans ce domaine.

Le sang menstruez.

Est-il prolongement du sang de l'accouchement, sang fécond, ou son contraire, sang stérile ? Lui aussi est chargé d'interdits, dangereux. A 1'Ehunya, par exemple, les femmes qui ont leurs règles peuvent assister aux sacrifices, mais en restant en retrait ; elles ne doivent surtout pas approcher la natte de la prêtresse, cette dernière ne pouvant pas non plus sacrifier pendant ses règles. Est-ce là le signe des vertus ambivalentes du sang des menstrues, mêlant "en même place le meilleur et le pire" ( $1 \%$ ). Comme le sang de l'accouchement, il risque d'inhiber par son contact, sa vue ou sa seule présence, les forces viriles. On pourrait être tenté d'assimiler les menstrues à un sacrifice renouvelé de la femme, allant dans le sens donc d'une libération d'énergie vitale. Ou bien faut-il l'opposer beaucoup plus nettement au sang de la naissance?

Comment dans ce champ situer le sang de la circoncision ? Ici ce qui, semble-t-il, importe avant tout dans l'opération, c'est qu'"un peu de sang coule" : les biola pratiquaient autrefois une simple incision, toujours au sanctuaire duboekin. Au regard des épreuves infligées à l'initié, de ces rites qui, partout, réactualisent le schéma mortrenaissance, n'est-ce-pas que tout passage initiatique suppose sacrifice de l'initié lui-même?

Il reste que, dans l'ensemble, les cérémonies aux ukin tenus par les femmes ont plus recours aux libations et offrandes vêgétales qu'aux sacrifices sanglants, voire s'en passent fort bien, tout en possédant la même efficace : l'explication par une "participation naturelle" des

17. Cf. M. GRIAULE, Dieu d'eau, 1947 ("Vingt-deuxième journée : le sang des femmes, et le battage de la digitaria"). 
femmes à l'acte sacrificiel, participation que libations et offrandes permettraient de réactualiser, prolonger, est tentante : mais que $11 \mathrm{e}$ place lui accorder, par rapport au fait que, le plus souvent, les gestionnaires des troupeaux familiaux, donc les pourvoyeurs en victimes animales (pour le gros bétail du moins), soient les hommes ?

Revenons peut-être sur certains de ces rites féminins non sacrificiels, dont l'objet pourtant peut être fondamental pour la survie du groupe : c'est le cas des rites accomplis en cas de sécheresse. Tous les $u k i n$ féminins ont un pouvoir plus ou moins important sur la pluie. Lorsque la pluie tarde à tomber, plusieurs recours sont possibles : de 1 'ordre du sacrifice à certains ukin, tel que celui du roi des pluies, du village d'Enampore, de Husila (boekin d'"appel à la tornade"), pour lesqueis on choisira de préférence des taureaux noirs. Mais aussi d'un autre ordre, tel que le nyukul-emit, rite général à tout le pays et beaucoup plus ancien que le culte du Husiza par exemple. Le rite est décidé par les fenmes de l'Ehunya réunies au sanctuaire, et c'est pratiquement le seul où Ata-emit (Emitay) soit directement interpellé, et lors duquel le sacrifice ne tient pas la place centrale. Très tôt le matin, la responsable de l'association part dans les rizières, où elle adresse un chant à Emitay, repris de loin en loin par toutes les femmes, qui organisent alors une procession à travers villages et rizières. Calebasses d'eau sur la tête et branchages feuillus en main, elles se rendent à l'Ehumya, où elles procèdent à des libations d'eau essentiellement, puis à un certain nombre d'autres ukin, dont elles avertissent les desservants (s'il s'agit d'ukin tenus par les hommes). Un certain nombre vont passer toutes leurs nuits dans le sanctuaire, durant la semaine où se déroulent les processions, tous travaux cessants. Dans la région du roi des pluies d'Enampore, la même cérémonie se déroule à l'un des ukin royaux, où le roi a auparavant sacrifié un taureau noir. Mais ici le roi ne peut agir sans le groupe féminin.

De fait, ce rite retient l'attention quant au rôle ici attribué aux femmes, qui interviennent de leur propre initiative, sans référence au côté masculin, et sans médiat sacrificiel. L'on pourrait d'ailleurs s'interroger sur la dimension politique de cette possibilité d'action directe sur la pluie, et sur ses ambiguités, puisqu'elles peuvent aussi supporter seules la responsabilité de la sécheresse. Responsabilité d'une autre nature que celle des hommes par exemple, qui refuseraient 
de prélever sur leurs biens pour sacrifier.

D'autres rites Eéminins posent la même question : que ce soient les danses dans les rizières, destinées à favoriser la germination, ou encore ces plongées collectives des femmes, nues le plus souvent, dans les marigots, en vue d'êloigner mauvais esprits, maladies, et autres personnages malfaisants qui siègent dans 1 'eau. Là encore, i1 est difficile d'assimiler simplement ces rites à des purifications. Les femmes doivent faire êcran au danger, l'eloigner par ces actions qui, associées ou non au sacrifice, ont en tout cas la même efficience. Au-delà du symbolisme très général quj. associe ferme et humidité, que tirer de ces rites, au regard des processus de réquilibration de forces, déclenchê lors du sacrifice ? D'où les femmes tirent-elles ce pouvoir d'ècarter les menaces qui. pèsent sur la collectivitê ou sur leurs enfants ?

A un niveau plus individuel, par exemple en cas de stérilité, on retrouve cette dualité de pratiques sacrificielles et non-sacrificielles, les secondes réalisées au travers d'un rituel très long, marqué par la séparation brutale de la femre d'avec les siens, d'avec son village, son changement de nom et son "adoption" par les sociétés de jeunes, et les femmes d'un autre villages : c'est le kanyalen cou kangaleri).

Au travers de ces indications et de ces interrogations, on peut tenter de repérer un certain nombre de convergences et de divergences d'avec le schéma proposé par Hubert et Mauss :

- d'une façon très gênérale, dans le sacrifice diola, il s'agit bien de modifier l'état de celui ou celle qui l'accomplit, ou de certains objets auxquels il s'intéresse. Mais le processus mis en oeuvre ici est bien plutôt pensé comme un processus d'échange de forces vitales, que conme un processus de "consécration/désécration", ou d'acquisition d"esprit".

-- le contact avec ces forces, ou plutôt leur mise en mouvement, est redoutable: une fois dêclenché, la continuité du sacrifice est requise. Mais le sacrifiant diola ne passe pas par ces précautions rituelles, voire ce rite de passage, dont parlent Hubert et Mauss; cela vaudrait bien plutôt pour le sacrificateur - au moment de son intronisation en particulier.

- on retrouve aussi dans l'exemple diola ce balancement entre 
rites de "conjonction" (plutôt que de sacralisation) (18), avec communion après le sacrifice, et rites de disjonction (sacrifice au boekin royal Jananande, sortie de 1a maternité, si tant est qu'on puisse rapprocher 1'accouchement du schêma sacrificiel...).

-- il paraît difficile, par contre, de parler à proprement dit de sacrifice d"expiation". L'expiation ici, c'est plutôt la maladie qui vous frappe quand le boekin "vous a attrapé", et le sacrifice intervient pour combler ce défaut d'ênergie vitale. De même, l'idée d'un transfert du sacrifiant à la victime n'est pas évidente.

-- les ukin diola ont, à I'évidence, besoin de profanes, c'est bien par le sacrifice qu'ils entretiennent leur existence et leur puissance.

Un point que je voudrais soulever pour terminer, c'est celui des raisons et des implications de ce prélèvement souvent massif sur des richesses qu'en tout autre moment les Diola tiennent soigneusement cachées des regards et des envies. Cette fonction sociale qu'assure le sacrifice par le renouvellement des liens d'alliance et de bonne entente inter- ou intra-concessions, entre quartiers et villages, par le rêtablissement d'équilibres troublès, n'est peut-être pas seulement célébration de la sacralité de la société, comme le postulent Hubert et Mauss.

La mise en scène périodique des richesses et de leur destruction, qui, pour le bétail, prend souvent 1'allure d'hécatombes, est aussi un moyen à la fois de signifier des inégalités de pouvoir économique et de les nier, dans la redistribution et la dilapidation des produits d'une accumulation, qui, malgré un système de production que 1 'on pourrait qualifier de "communautaire", apparaît inégale.

Dans quelle mesure, à ce moment-là, les effets politiques et religieux du sacrifice ne-se trouvent-ils pas parfois "court-circuités" par ces pratiques féminines auxquelles j'ai fait allusion, moins coûteuses en biens, mais peut-être plus coíteuses socialement, lorsqu'éclatent aux yeux des hommes les limites de leurs pouvoirs.

18. Cf. L. de Heusch, op. cit. Le sacrifice 1. 